\title{
An Efficiency Measure for Dynamic Networks Modeled as Evolutionary Variational Inequalities with Application to the Internet \\ and
}

Vulnerability Analysis

\author{
Anna Nagurney and Qiang Qiang \\ Department of Finance and Operations Management \\ Isenberg School of Management \\ University of Massachusetts \\ Amherst, Massachusetts 01003
}

January 2007; revised August 2007; January 2008

Netnomics 9 (2008), pp. 1-20.

\begin{abstract}
In this paper, we propose an efficiency/performance measure for dynamic networks, which have been modeled as evolutionary variational inequalities. Such applications include the Internet. The measure, which captures demands, flows, and costs/latencies over time, allows for the identification of the importance of the nodes and links and their rankings. We provide both continuous time and discrete time versions of the efficiency measure. We illustrate the efficiency measure for the time-dependent (demand-varying) Braess paradox and demonstrate how it can be used to assess the most vulnerable nodes and links in terms of the greatest impact of their removal on the efficiency/performance of the dynamic network over time.
\end{abstract}

Keywords: network efficiency, performance measurement, dynamic networks, Internet, Braess paradox, vulnerability analysis, critical network components, evolutionary variational inequalities 


\section{Introduction}

Networks provide the foundations for communication, transportation and logistics, energy provision, social interactions, as well as financing. The study of networks spans many disciplines due to their wide application and importance. Indeed, today, the subject has garnered renewed interest, since a spectrum of catastrophic events such as $9 / 11$, the North American electric power blackout of 2003, as well as Hurricane Katrina in 2005, among others, have reinforced our need for and dependence on network systems (see, e.g., Nagurney $(2006))$.

The Internet, in particular, as a telecommunications network, and with linkages to other network systems, including transportation and financial ones, as well as energy systems, is fundamental to the functioning of our modern societies and economies. Hence, an appropriate efficiency measure and a means of identifying its critical nodes and links is essential to not only the management of such a dynamic network but also to its very security. Clearly, those nodes and links that are deemed most important are those that also merit greatest protection.

In this paper, we propose a network efficiency measure for dynamic networks, which have been modeled as evolutionary variational inequalities. Such dynamic networks include the Internet (cf. Nagurney, Parkes, and Daniele (2007)). To-date, the network efficiency measure proposed by Latora and Marchiori (2001), which is the sum of the inverses of the shortest paths based on geodesic distances between nodes, multiplied by one over the number of nodal pairs, has been applied to several network systems, including the MBTA subway system in Boston and the Internet (cf. Latora and Marchiori (2003, 2004)). However, their measure does not include behavior that would be associated with rerouting in the case of nodal or link failures nor does it explicitly include traffic flows. Furthermore, it does not capture the time dimension. Bienenstock and Bonacich (2003), in turn, focused on social networks and their efficiency versus vulnerability and proposed a network efficiency measure, which actually coincides with that of Latora and Marchiori (2001). More recently, Jenelius, Petersen, and Mattsson (2006) proposed a network efficiency measure for congested transportation networks but the measure requires that the network remain connected after disruptions. Furthermore, it does not measure the usage of the network as demand varies 
over time.

We use as the basis for our dynamic network measurement framework the time-dependent, evolutionary variational inequality (EVI) model of the Internet proposed by Nagurney, Parkes, and Daniele (2007). Indeed, since the demand for Internet resources itself is dynamic, an underpinning modeling framework must be able to handle time-dependent constraints. Roughgarden (2005) on page 10 notes that, "A network like the Internet is volatile. Its traffic patterns can change quickly and dramatically ... The assumption of a static model is therefore particularly suspect in such networks."

It has been shown (cf. Roughgarden (2005) and the references therein) that distributed routing, which is common in computer networks and, in particular, the Internet, and "selfish" (or "source" routing in computer networks) routing, as occurs in the case of user-optimized transportation networks, in which travelers select the minimum cost route between an origin and destination, are one and the same if the cost functions associated with the links that make up the paths/routes coincide with the lengths used to define the shortest paths. In this paper, we assume that the costs on the links are congestion-dependent, that is, they depend on the volume of the flow on the link. Note that network efficiency measurements based on shortest path geodesic distances ignore this property of congested networks. We permit the cost on a link to represent travel delay but we utilize "cost" functions since these are more general conceptually than delay (or latency) functions and they can include, for example, tolls associated with pricing, etc.

In addition, we emphasize that, in the case of transportation networks, it is travelers that make the decisions as to the route selection between origin/destination (O/D) pairs of nodes, whereas in the case of the Internet, it is algorithms, implemented in software, that determine the shortest paths. Furthermore, we mention that analogues exist between transportation networks and telecommunication networks and, in particular, the Internet, in terms of decentralized decision-making, flows and costs, and even the Braess paradox, which allow us to take advantage of such a connection (cf. Beckmann, McGuire, and Winsten (1956), Beckmann (1967), Braess (1968), Dafermos and Sparrow (1969), Cantor and Gerla (1974), Gallager (1977), Bertsekas and Tsitsiklis (1989), Bertsekas and Gallager (1992), Ran and Boyce (1996), Korilis, Lazar, and Orda (1999), Boyce, Mahmassani, and Nagurney 
(2005), Resende and Pardalos (2006), Nagurney, Parkes, and Daniele (2007)).

The evolutionary variational inequality methodology that Nagurney, Parkes, and Daniele (2007) utilized for the formulation and analysis of the Internet is quite natural for several reasons. First, historically, finite-dimensional variational inequality theory (cf. Smith (1979), Dafermos (1980), Nagurney (1993), Patriksson (1994), and the references therein) has been used to generalize static transportation network equilibrium models dating to the classic work of Beckmann, McGuire, and Winsten (1956), which also forms the foundation for selfish routing and decentralized decision-making on the Internet (see, e.g., Roughgarden (2005)). Secondly, there has been much research activity devoted to the development of models for dynamic transportation problems and it makes sense to exploit the connections between transportation networks and the Internet (see also Nagurney and Dong (2002) and Boyce, Mahmassani, and Nagurney (2005)). In addition, EVIs, which are infinitedimensional, have been used to model a variety of time-dependent applications, including time-dependent spatial price problems, financial network problems, dynamic supply chains, and electric power networks (cf. Daniele, Maugeri, and Oettli (1999), Daniele (2003, 2004), Daniele (2006), Nagurney et al. (2007), and Nagurney (2006)). Hence, we believe that they are a rather natural formalism for dynamic networks and the measurement of their efficiency/performance.

The structure of the paper is as follows. In Section 2 we briefly recall the evolutionary variational inequality formulation of the Internet due to Nagurney, Parkes, and Daniele (2007). In Section 3, we propose the efficiency measure for dynamic networks in both continuous time and discrete time forms. In Section 4, we apply the measure to the Braess paradox network with time-varying demands to determine the network efficiency as well as the importance rankings of the nodes and links. In Section 5, we summarize the results in this paper and present our conclusions. 


\section{Evolutionary Variational Inequalities and the Internet}

For definiteness, in this section, we review the evolutionary variational inequality model of the Internet, recently proposed by Nagurney, Parkes, and Daniele (2007), but, for simplicity, we consider the single class, rather than the multiclass version.

In particular, the Internet, a dynamic network par excellence, is modeled as a network $G=[N, L]$, consisting of the set of nodes $N$ and the set of directed links $L$. The set of links $L$ consists of $n_{L}$ elements. The set of O/D pairs of nodes is denoted by $W$ and consists of $n_{W}$ elements. We denote the set of routes (with a route consisting of links) joining the O/D pair $w$ by $P_{w}$. We assume that the routes are acyclic. Let $P$ with $n_{P}$ elements denote the set of all routes connecting all the $\mathrm{O} / \mathrm{D}$ pairs in the Internet. Links are denoted by $a, b$, etc; routes by $r, q$, etc., and $\mathrm{O} / \mathrm{D}$ pairs by $w_{1}, w_{2}$, etc. We assume that the Internet is traversed by a single class of "job" or "task."

Let $d_{w}(t)$ denote the demand, that is, the traffic generated, between $\mathrm{O} / \mathrm{D}$ pair $w$ at time $t$. The flow on route $r$ at time $t$, which is assumed to be nonnegative, is denoted by $x_{r}(t)$ and the flow on link $a$ at time $t$ by $f_{a}(t)$.

Since the demands over time are assumed known, the following conservation of flow equations must be satisfied at each $t$ :

$$
d_{w}(t)=\sum_{r \in P_{w}} x_{r}(t), \quad \forall w \in W,
$$

that is, the demand associated with an O/D pair must be equal to the sum of the flows on the routes that connect that $\mathrm{O} / \mathrm{D}$ pair. Also, we must have that

$$
0 \leq x_{r}(t) \leq \mu_{r}(t), \quad \forall r \in P,
$$

where $\mu_{r}(t)$ denotes the capacity on route $r$ at time $t$.

We group the demands at time $t$ for all the $\mathrm{O} / \mathrm{D}$ pairs into the $n_{W}$-dimensional vector $d(t)$ and the route flows at time $t$ into the $n_{P}$-dimensional vector $x(t)$. The upper bounds/capacities on the routes at time $t$ are grouped into the $n_{P}$-dimensional vector $\mu(t)$.

The link flows are related to the route flows, in turn, through the following conservation 
of flow equations:

$$
f_{a}(t)=\sum_{r \in P} x_{r}(t) \delta_{a r}, \quad \forall a \in L
$$

where $\delta_{a r}=1$ if link $a$ is contained in route $r$, and $\delta_{a r}=0$, otherwise. Hence, the flow on a link is equal to the sum of the flows on routes that contain that link. All the link flows at time $t$ are grouped into the vector $f(t)$, which is of dimension $n_{L}$.

The cost on route $r$ at time $t$ is denoted by $C_{r}(t)$ and the cost on a link $a$ at time $t$ by $c_{a}(t)$. We allow the cost on a link, in general, to depend upon the entire vector of link flows at time $t$, so that

$$
c_{a}(t)=c_{a}(f(t)), \quad \forall a \in L
$$

In view of (3), we may write the link costs as a function of route flows, that is,

$$
c_{a}(x(t)) \equiv c_{a}(f(t)), \quad \forall a \in L
$$

The costs on routes are related to costs on links through the following equations:

$$
C_{r}(x(t))=\sum_{a \in L} c_{a}(x(t)) \delta_{a r}, \quad \forall r \in P
$$

which means that the cost on a route at a time $t$ is equal to the sum of costs on links that make up the route at time $t$. We group the route costs at time $t$ into the vector $C(t)$, which is of dimension $n_{P}$.

We now define the feasible set $\mathcal{K}$. We consider the Hilbert space $\mathcal{L}=L^{2}\left([0, T], R^{n_{P}}\right)$ (where $T$ denotes the time interval under consideration) given by

$\mathcal{K}=\left\{x \in L^{2}\left([0, T], R^{n_{P}}\right): 0 \leq x(t) \leq \mu(t)\right.$ a.e. in $[0, T] ; \sum_{p \in P_{w}} x_{p}(t)=d_{w}(t), \forall w$, a.e. in $\left.[0, T]\right\}$.

We assume that the capacities $\mu_{r}(t)$, for all $r$, are in $\mathcal{L}$ and that the demands, $d_{w} \geq 0$, for all $w$, are also in $\mathcal{L}$. Further, we assume that

$$
0 \leq d(t) \leq \Phi \mu(t) \text {, a.e. on }[0, T]
$$


where $\Phi$ is the $n_{W} \times n_{P}$-dimensional $\mathrm{O} / \mathrm{D}$ pair-route incidence matrix, with element $(w, r)$ equal to 1 if route $r$ is contained in $P_{w}$, and 0 , otherwise. Due to (8), the feasible set $\mathcal{K}$ is nonempty. As noted in Nagurney, Parkes, and Daniele (2007), $\mathcal{K}$ is also convex, closed, and bounded. Note that we are not restricted as to the form that the time-varying demands for the $\mathrm{O} / \mathrm{D}$ pairs take since convexity is guaranteed even if the demands have a step-wise structure, or are piecewise continuous.

The dual space of $\mathcal{L}$ is denoted by $\mathcal{L}^{*}$. On $\mathcal{L} \times \mathcal{L}^{*}$ we define the canonical bilinear form by

$$
\langle\langle\mathcal{G}, x\rangle\rangle:=\int_{0}^{T}\langle\mathcal{G}(t), x(t)\rangle d t, \quad \mathcal{G} \in \mathcal{L}^{*}, \quad x \in \mathcal{L}
$$

Furthermore, the cost mapping $C: \mathcal{K} \rightarrow \mathcal{L}^{*}$, assigns to each flow trajectory $x(\cdot) \in \mathcal{K}$ the cost trajectory $C(x(\cdot)) \in \mathcal{L}^{*}$.

We now state the dynamic network equilibrium conditions governing the Internet, assuming shortest path routing. These conditions are a generalization of the Wardropian (1952) first principle of traffic behavior (see also, e.g., Beckmann, McGuire, and Winsten (1956), Dafermos and Sparrow (1969), Dafermos (1980), and Nagurney (1993)) to include the time dimension and capacities on the route flows. Of course, if the capacities are very large and exceed the demand at each $t$, then the upper bounds are never attained by the route flows and the conditions below will collapse, in the case of fixed time $t$, to the well-known static network equilibrium conditions (see Dafermos and Sparrow (1969), Dafermos (1980), and the references therein). The below is a special case of the multiclass network equilibrium conditions stated in Nagurney, Parkes, and Daniele (2007); see also Daniele (2006).

\section{Definition 1: Dynamic Network Equilibrium}

A route flow pattern $x^{*} \in \mathcal{K}$ is said to be a dynamic network equilibrium (according to the generalization of Wardrop's first principle), if, at each time $t$, only the minimum cost routes not at their capacities are used (that is, have positive flow) for each $O / D$ pair unless the flow on a route is at its upper bound (in which case those routes' costs can be lower than those on the routes not at their capacities). The state can be expressed by the following equilibrium conditions which must hold for every $O / D$ pair $w \in W$, every route $r \in P_{w}$, and a.e. on 
$[0, T]:$

$$
C_{r}\left(x^{*}(t)\right)-\lambda_{w}^{*}(t)\left\{\begin{array}{lll}
\leq 0, & \text { if } & x_{r}^{*}(t)=\mu_{r}(t), \\
=0, & \text { if } & 0<x_{r}^{*}(t)<\mu_{r}(t), \\
\geq 0, & \text { if } & x_{r}^{*}(t)=0 .
\end{array}\right.
$$

Hence, conditions (10) state that all utilized routes not at their capacities connecting an $\mathrm{O} / \mathrm{D}$ pair have equal and minimal costs at each time $t$ in $[0, T]$. If a route flow is at its capacity then its cost can be lower than the minimal cost for that O/D pair. Of course, if we have that $\mu_{r}=\infty$, for all routes $r \in P$, then the dynamic equilibrium conditions state that all used routes connecting an $\mathrm{O} / \mathrm{D}$ pair of nodes have equal and minimal route costs at each time $t$. For fixed $t$, the latter conditions coincide with a single class version of Wardrop's first principle (see Dafermos and Sparrow (1969) governing static transportation network equilibrium problems). Note that this concept has also been applied to static models of the Internet (cf. Roughgarden (2005) and the references therein).

In the above discussion, we assume that the general "cost" on links and paths is "instantaneous," which solely depends on the current network flows and demands. Such an assumption is relatively general and can be easily extended to the discrete-time case, which will be discussed in the following section. Indeed, in the discrete-time case, demand is assumed to be constant during each time interval and, therefore, the link and path costs can be interpreted as the average costs based on the flows in that particular time interval. Furthermore, similar data and computer communication network models and analysis, but in the discrete-time case, have also been studied by Bertsekas and Gallager (1992).

The standard form of the EVI that we work with is:

$$
\text { determine } x^{*} \in \mathcal{K} \text { such that }\left\langle\left\langle F\left(x^{*}\right), x-x^{*}\right\rangle\right\rangle \geq 0, \forall x \in \mathcal{K} \text {. }
$$

We now state the following theorem, which is an adaptation of Theorem 1 in Nagurney, Parkes, and Daniele (2007) to the single-class case; see that reference for a proof. 


\section{Theorem 1}

$x^{*} \in \mathcal{K}$ is an equilibrium flow according to Definition 1 if and only if it satisfies the evolutionary variational inequality:

$$
\int_{0}^{T}\left\langle C\left(x^{*}(t)\right), x(t)-x^{*}(t)\right\rangle d t \geq 0, \quad \forall x \in \mathcal{K} .
$$

Furthermore, for completeness, we also provide some existence results:

\section{Theorem 2 (cf. Daniele, Maugeri, and Oettli (1999) and Daniele (2006))}

If $C$ in (12) satisfies any of the following conditions:

1. $C$ is hemicontinuous with respect to the strong topology on $\mathcal{K}$, and there exist $A \subseteq \mathcal{K}$ nonempty, compact, and $B \subseteq \mathcal{K}$ compact such that, for every $y \in \mathcal{K} \backslash A$, there exists $x \in B$ with $\langle\langle C(x), y-x\rangle\rangle<0$;

2. $C$ is hemicontinuous with respect to the weak topology on $\mathcal{K}$;

3. $C$ is pseudomonotone and hemicontinuous along line segments,

then the EVI problem (12) admits a solution over the constraint set $\mathcal{K}$.

Recall that $C: \rightarrow \mathcal{L}^{*}$, where $\mathcal{K}$ is convex, is said to be

pseudomonotone if and only if, for all $x, y \in \mathcal{K}$

$$
\langle\langle C(x), y-x\rangle\rangle \leq 0 \Rightarrow\langle\langle C(y), x-y\rangle\rangle \leq 0
$$

hemicontinuous if and only if, for all $y \in \mathcal{K}$, the function $\xi \mapsto\langle\langle C(\xi), y-\xi\rangle\rangle$ is upper semicontinuous on $\mathcal{K}$;

hemicontinuous along line segments if and only if, for all $x, y \in \mathcal{K}$, the function $\xi \mapsto$ $\langle\langle C(\xi), y-x\rangle\rangle$ is upper semicontinuous on the line segment $[x, y]$. 
Moreover, if $C$ is strictly monotone, then the solution of (12) is unique (see, e.g., Kinderlehrer and Stampacchia (1980)).

Daniele, Maugeri, and Oettli (1999) presented dynamic network equilibrium conditions for transportation networks. Here, we state the dynamic equilibrium conditions in a manner that is more transparent (cf. (10)), noting that the lower bounds on the route flows on the Internet will be zero.

We note that the solution of finite-dimensional variational inequalities is at quite an advanced state (cf. Nagurney (1993)). The solution of evolutionary variational inequalities, which are infinite-dimensional, is a topic discussed in the books by Daniele (2006) and Nagurney (2006) as well as in the papers by Cojocaru, Daniele, and Nagurney $(2005,2006)$ and in Barbagallo (2007). In particular, under certain conditions, an evolutionary variational inequality of the form (12) can be solved at discrete points in time and the solutions then interpolated. Hence, the advances in the computation of finite-dimensional variational inequalities, with many network equilibrium problems solved to-date, may be exploited for the effective solution of the EVI for dynamic networks.

We now present a small dynamic network numerical example.

\section{Example 1: A Simple Numerical Example}

Consider a network (small subnetwork of the Internet) consisting of two nodes and two links as in Figure 1. Since the routes consist of single links we work with the routes directly as in Figure 1. The route costs are:

$$
C_{r_{1}}(x(t))=2 x_{r_{1}}(t)+5, \quad C_{r_{2}}(x(t))=2 x_{r_{2}}(t)+10 .
$$

Also, we assume that the route capacities are: $\mu_{r_{1}}(t)=\mu_{r_{2}}(t)=\infty$.

The time horizon is $[0,10]$. There is a single $\mathrm{O} / \mathrm{D}$ pair $w=(1,2)$ and the time-varying demand is assumed to be $d_{w}(t)=t$.

Given the special structure of the network in Figure 1 and the linearity and separability of the route cost functions, it is easy to determine the equilibrium route trajectories satisfying (10), or, equivalently, (12), and they are: for $t \in\left[0,2 \frac{1}{2}\right], x_{r_{1}}^{*}(t)=t$ and $x_{r_{2}}^{*}(t)=0$, and for 


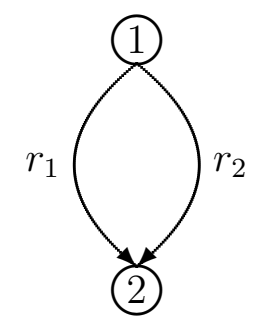

Figure 1: Network Structure of Example 1

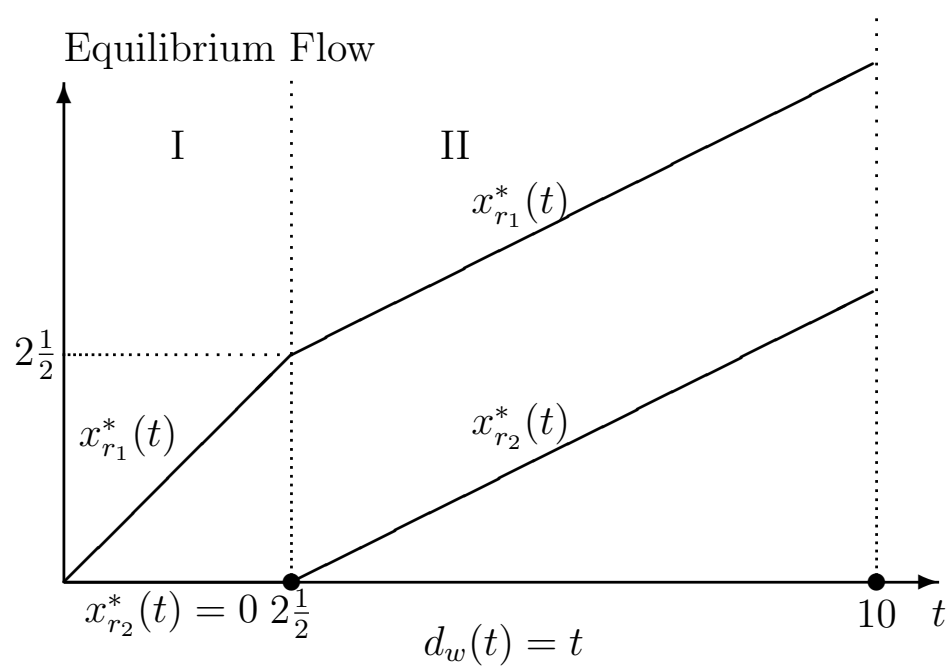

Figure 2: Equilibrium Trajectories of the Simple Numerical Example with Time-Dependent Demands

$t \in\left[2 \frac{1}{2}, 10\right], x_{r_{1}}^{*}(t)=\frac{1}{2} t+1 \frac{1}{4}$ and $x_{r_{2}}^{*}(t)=\frac{1}{2} t-1 \frac{1}{4}$.

The equilibrium flow trajectories of the above network are depicted in Figure 2.

\section{The Efficiency Measure for Dynamic Networks Modeled as Evolutionary Variational Inequalities}

In this section, we propose an efficiency measure for dynamic networks, modeled as evolutionary variational inequalities, which we denote by $\mathcal{E}(G, d, T)$.

\section{Definition 2a: Dynamic Network Efficiency: Continuous Time Version}

The network efficiency for the network $G$ with time-varying demand $d$ for $t \in[0, T]$, denoted 
by $\mathcal{E}(G, d, T)$, is defined as follows:

$$
\mathcal{E}(G, d, T)=\frac{\int_{0}^{T}\left[\sum_{w \in W} \frac{d_{w}(t)}{\lambda_{w}(t)}\right] / n_{W} d t}{T} .
$$

The dynamic network efficiency measure $\mathcal{E}$ defined in (13) is actually the average demand to price ratio over time. It measures the overall (economic) functionality of the network with time-varying demands. When the network topology $G$, the demand pattern over time and the time span are given, a network is considered to be more efficient if it can satisfy higher demands at lower costs over time.

The network efficiency measure (13) can be easily adapted to dynamic networks in which the demands change at discrete points in time, as we now demonstrate. Let $d_{w}^{1}, d_{w}^{2}, \ldots, d_{w}^{H}$ denote demands for $\mathrm{O} / \mathrm{D}$ pair $w$ in $H$ discrete time intervals, given, respectively, by:

$\left[t_{0}, t_{1}\right],\left(t_{1}, t_{2}\right], \ldots,\left(t_{H-1}, t_{H}\right]$, where $t_{H} \equiv T$. We assume that the demand is constant in each such time interval for each $\mathrm{O} / \mathrm{D}$ pair. Moreover, we denote the corresponding minimal costs for each $\mathrm{O} / \mathrm{D}$ pair $w($ see $(10))$ at the $H$ different time intervals by: $\lambda_{w}^{1}, \lambda_{w}^{2}, \ldots, \lambda_{w}^{H}$. The demand vector $d$, in this special discrete case, is a vector in $R^{n_{W} \times H}$. The dynamic network efficiency measure in this case is as follows:

\section{Definition 2b: Dynamic Network Efficiency: Discrete Time Version}

The network efficiency for the network $(G, d)$ over $H$ discrete time intervals: $\left[t_{0}, t_{1}\right],\left(t_{1}, t_{2}\right], \ldots,\left(t_{H-1}, t_{H}\right]$, where $t_{H} \equiv T$, and with the respective constant demands: $d_{w}^{1}, d_{w}^{2}, \ldots, d_{w}^{H}$ for all $w \in W$ is defined as follows:

$$
\mathcal{E}\left(G, d, t_{H}=T\right)=\frac{\sum_{i=1}^{H}\left[\left(\sum_{w \in W} \frac{d_{w}^{i}}{\lambda_{w}^{i}}\right)\left(t_{i}-t_{i-1}\right) / n_{W}\right]}{t_{H}} .
$$

We now provide the relationship between the dynamic network efficiency measure (13) and the network efficiency measure proposed by Nagurney and Qiang (2007a,b) for static transportation (or congested) networks with fixed demands. 


\section{Theorem 3}

Assume that $d_{w}(t)=d_{w}$, for all $O / D$ pairs $w \in W$ and for $t \in[0, T]$. Then, the dynamic network efficiency measure (13) collapses to the Nagurney and Qiang (2007a, b) measure:

$$
\mathcal{E}=\frac{1}{n_{W}} \sum_{w \in W} \frac{d_{w}}{\lambda_{w}}
$$

Proof: Since the $d_{w}$ 's are fixed over the time horizon $[0, T]$, the minimal equilibrium route costs for each O/D pair, denoted by $\lambda_{w}, \forall w \in W$, are also fixed over the time horizon. Substituting these terms into (13), we obtain:

$$
\mathcal{E}(G, d, T)=\frac{\int_{0}^{T}\left[\sum_{w \in W} \frac{d_{w}}{\lambda_{w}}\right] / n_{W} d t}{T}=\frac{T}{T}\left[\sum_{w \in W} \frac{d_{w}}{\lambda_{w}}\right] / n_{W}=\left[\sum_{w \in W} \frac{d_{w}}{\lambda_{w}}\right] / n_{W} .
$$

But the right-most expression in (16) is precisely the Nagurney and Qiang (2007a, b) measure for static traffic network equilibrium problems.

Several points merit some discussion here. First, one of the advantages of the above measure is that it allows us to study the network efficiency where there exist disconnected $\mathrm{O} / \mathrm{D}$ pairs. Such a feature is extremely useful in assessing the network functionality under partial disruptions whereas traditional measures, such as the total network cost over time, are no longer applicable (cf. Roughgarden (2005)). An illustrative example will be given later in this section. Second, in the above definition, we consider the network model without explicit edge, that is, link, capacity constraints. It is well-known in the transportation literature that edge capacities can be implicitly included in the link travel cost functions in which case the travel time tends to infinity as the link flows approach their respective capacities (cf. Daganzo (1977a, b)). A network model with explicit edge capacity constraints was studied by Patriksson (1994), who generalized link costs to reformulate the network as an equivalent uncapacitated network. Hence, in such a model, $\lambda_{w}(t)$ in (13) can be interpreted as a generalized cost. Nevertheless, a discussion of the distinction and the relationships between the two approaches is not the focus of the current paper. We will devote our future research to the network model with explicit edge capacity constraints. 
The importance of a network component in the dynamic network case is the same as that defined in Nagurney and Qiang (2007a, b), but with the static efficiency measure now replaced by the dynamic network efficiency measure given by (13) in the continuous case and by (14) in the discrete case. Hence, we have the following:

\section{Definition 3: Importance of a Network Component}

The importance of network component $g$ of network $G$ with demand d over time horizon $T$ is defined as follows:

$$
I(g, d, T)=\frac{\mathcal{E}(G, d, T)-\mathcal{E}(G-g, d, T)}{\mathcal{E}(G, d, T)}
$$

where $\mathcal{E}(G-g, d, T)$ is the dynamic network efficiency after component $g$ is removed.

In studying the importance of a network component, the elimination of a link is treated in the above measure by removing that link while the removal of a node is managed by removing the links entering and exiting that node. In the case that the removal results in no path/route connecting an O/D pair, we simply assign the demand for that O/D pair to an abstract path with a cost of infinity. Hence, our measure is well-defined even in the case of disconnected networks; see also Nagurney and Qiang (2007a, b). Additional theoretical properties of the static measure can be found in Qiang and Nagurney (2008).

For illustration purpose, we apply the above dynamic network efficiency measure to study the importance of links and nodes in Example 1 in Section 2. For the time horizon given by $t \in[0,10]$, with $T=10$, the network efficiency $\mathcal{E}(G, d, 10)=0.3686$. The importance and the rankings of the links and the nodes are given in Tables 1 and 2, respectively.

Table 1: Importance and Ranking of Links in Example 1

\begin{tabular}{|c|c|c|}
\hline Link & Importance Value & Importance Ranking \\
\hline$r_{1}$ & 0.3887 & 1 \\
\hline$r_{2}$ & 0.1894 & 2 \\
\hline
\end{tabular}

It is quite reasonable that link/route $r_{1}$ is more important than link/route $r_{2}$, as reported in Table 1, since over the time horizon it carries more traffic. Note that a measure such as the 
Table 2: Importance and Ranking of Nodes in Example 1

\begin{tabular}{|c|c|c|}
\hline Node & Importance Value & Importance Ranking \\
\hline 1 & 1.0000 & 1 \\
\hline 2 & 1.0000 & 1 \\
\hline
\end{tabular}

Latora and Marchiori (2001) measure does not capture either flows or the time dimension.

Furthermore, as discussed previously in this section, the proposed dynamic network efficiency measure enables us to study the network with disconnected O/D pairs while the other measure (e.g. total network cost over time) is not applicable. The following simple example is given to illustrate this idea.

\section{Example 2: An Illustrative Network Example}

Consider the following network, as shown in Figure 3, with three nodes and two links. Nodes are denoted by 1,2 , and 3 , and links are denoted by $a$ and $b$. Similar to Example 1, since the routes consist of single links we work with the routes directly, so that $r_{1}=a$ and $r_{2}=b$. The route costs are:

$$
C_{r_{1}}(x(t))=x_{r_{1}}(t)+1, \quad C_{r_{2}}(x(t))=x_{r_{2}}(t)+2 .
$$

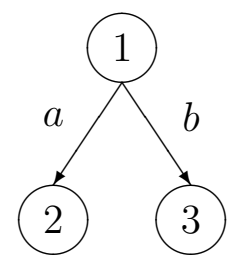

Figure 3: Network Structure of Example 2

Also, we assume that the route capacities are $\mu_{r_{1}}(t)=\mu_{r_{1}}(t)=\infty$.

The time horizon is $[0,10]$. There are two O/D pairs, namely, $w_{1}=(1,2)$ and $w_{2}=(1,3)$. The time-varying demands are assumed to be $d_{w_{1}}(t)=3 t+10$ and $d_{w_{2}}(t)=3 t$. 
Given the special structure of the network, it is easy to determine the equilibrium route trajectories satisfying (10), or equivalently, (12), and they are: $x_{r_{1}}^{*}(t)=3 t+10$ and $x_{r_{2}}^{*}(t)=$ $3 t$.

The network efficiency measure is given by $\mathcal{E}(G, d, 10)=0.8857$.

The importance and the rankings of the links and the nodes are given in Tables 3 and 4, respectively.

Table 3: Importance and Ranking of Links in Example 2

\begin{tabular}{|c|c|c|}
\hline Link & Importance Value & Importance Ranking \\
\hline$a$ & 0.5398 & 1 \\
\hline$b$ & 0.4602 & 2 \\
\hline
\end{tabular}

Table 4: Importance and Ranking of Nodes in Example 2

\begin{tabular}{|c|c|c|}
\hline Node & Importance Value & Importance Ranking \\
\hline 1 & 1.0000 & 1 \\
\hline 2 & 0.5398 & 2 \\
\hline 3 & 0.4602 & 3 \\
\hline
\end{tabular}

In the above example, link $a /$ route $r_{1}$ carries more flow over time and, therefore, it is ranked higher than link $b /$ route $\mathrm{r}_{2}$.

Moreover, the total cost of the network over time, after a link or a node is destroyed, becomes infinite in Example 2. Hence, such an undesirable feature prevents us from assessing the network efficiency under disruptions and, as a consequence, the importance and the rankings of the network components, under a total cost measure. In particular, when a disruption causes partial damage to a large network and a certain demand cannot be satisfied, the remaining network components still may function properly. Hence, it still makes sense to analyze network performance in such a situation and, obviously, those network components whose removal causes a large performance drop should receive more attention. Therefore, 
the proposed measure provides important insights into network vulnerability and security analysis.

\section{An Application to the Time-Dependent Braess Network}

In this Section, we apply the above proposed dynamic network measure to the timedependent Braess example (cf. Nagurney, Parkes, and Daniele (2007), Nagurney (2006), and, also, Pas and Principio (1997)), but, first, for completeness and easy reference, we revisit the Braess (1968) paradox (see also, Boyce, Mahmassani, and Nagurney (2005) and Braess, Nagurney, and Wakolbinger (2005)). Recall that in the Braess paradox, which is an example of a fixed demand network equilibrium problem, the addition of a new link, which yields a new route, makes all the "users" in the network worse off. As emphasized in Korilis, Lazar, and Orda (1999), this is also relevant to the Internet. We present an EVI formulation with instantaneous costs which deepens the understanding of the Braess paradox and also illustrates dramatically the importance of time-varying demands and the associated equilibrium flows and costs.

\section{The Time-Dependent Braess Paradox}

Assume a network as the first network depicted in Figure 4 in which there are four nodes: 1, 2, 3, 4; four links: $a, b, c, d$; and a single O/D pair $w=(1,4)$. There are, hence, two routes available between this O/D pair: $r_{1}=(a, c)$ and $r_{2}=(b, d)$.

The networks given in Figure 4 are due to Braess (1968). We now construct timedependent link costs, route costs, and demand for $t \in[0, T]$. It is important to emphasize that the case where time $t$ is discrete, that is, $t=0,1,2, \ldots, T$, is trivially included in the equilibrium conditions (10) and also captured in the EVI formulation (12).

We consider, to start, the first network in Figure 4, consisting of links: $a, b, c, d$. We assume that the capacities $\mu_{r_{1}}(t)=\mu_{r_{2}}(t)=\infty$ for all $t \in[0, T]$. The link cost functions are assumed to be given and as follows for time $t \in[0, T]$ :

$$
c_{a}\left(f_{a}(t)\right)=10 f_{a}(t), \quad c_{b}\left(f_{b}(t)\right)=f_{b}(t)+50, \quad c_{c}\left(f_{c}(t)\right)=f_{c}(t)+50, \quad c_{d}\left(f_{d}(t)\right)=10 f_{d}(t) .
$$

We assume a time-varying demand $d_{w}(t)=t$ for $t \in[0, T]$. 


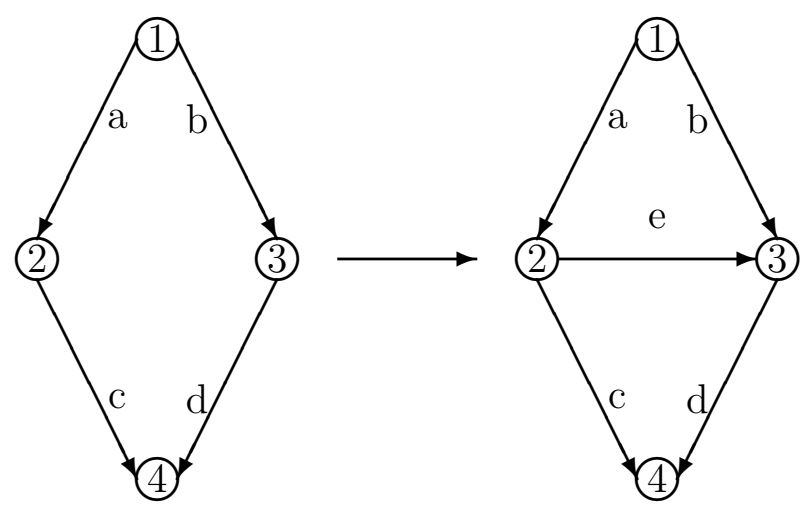

Figure 4: The Time-Dependent Braess Network Example with Relevance to the Internet

Observe that at time $t=6, d_{w}(6)=6$, and it is easy to verify that the equilibrium route flows at time $t=6$ are: $x_{r_{1}}^{*}(6)=3, \quad x_{r_{2}}^{*}(6)=3$, the equilibrium link flows are: $f_{a}^{*}(6)=3$, $f_{b}^{*}(6)=3, f_{c}^{*}(6)=3, \quad f_{d}^{*}(6)=3$, with associated equilibrium route costs:

$$
C_{r_{1}}(6)=c_{a}(6)+c_{c}(6)=83, \quad C_{r_{2}}=c_{b}(6)+c_{d}(6)=83,
$$

and, hence, equilibrium condition (10) is satisfied for time $t=6$. This is the solution to the classical (static) Braess (1968) network without the route addition.

We now construct and solve EVI (12) for the dynamic network equilibrium problem over $t \in[0, T]$. We first express the route costs in terms of route flows for Network 1 in Figure 4, where we have that, because of the conservation of flow equations $(3), f_{a}(t)=f_{c}(t)=x_{r_{1}}(t)$ and $f_{b}(t)=f_{d}(t)=x_{r_{2}}(t)$. That is, we must have that $C_{r_{1}}(t)=11 x_{r_{1}}(t)+50, C_{r_{2}}(t)=$ $11 x_{r_{2}}(t)+50$, with the route conservation of flow equations (1) yielding: $d_{w}(t)=t=$ $x_{r_{1}}(t)+x_{r_{2}}(t)$, and, hence, we may write $x_{r_{2}}(t)=t-x_{r_{1}}(t)$.

Similarly, we must have, because of the feasible set $\mathcal{K}$ (cf. (7)), the simplicity of the network topology, and the cost structure, that

$$
x_{r_{1}}^{*}(t)=x_{r_{2}}^{*}(t) .
$$

Hence, we may write EVI (12) for this problem as: determine $x^{*} \in \mathcal{K}$ satisfying

$$
\int_{0}^{T}\left(11 x_{r_{1}}^{*}(t)+50\right) \times\left(x_{r_{1}}(t)-x_{r_{1}}^{*}(t)\right)+\left(11 x_{r_{1}}^{*}(t)+50\right) \times\left(x_{r_{2}}(t)-x_{r_{2}}^{*}(t)\right) d t \geq 0, \quad \forall x \in \mathcal{K},
$$


which, in view of the feasibility condition: $x_{r_{1}}(t)+x_{r_{2}}(t)=t$, implies that (19) can be expressed as:

$$
\int_{0}^{T}\left(11 x_{r_{1}}^{*}(t)+50\right) \times\left(x_{r_{1}}(t)-x_{r_{1}}^{*}(t)\right)+\left(11 x_{r_{1}}^{*}(t)+50\right) \times\left(t-x_{r_{1}}(t)-x_{r_{1}}^{*}(t)\right) d t \geq 0, \quad \forall x \in \mathcal{K},
$$

which, after algebraic simplification, is

$$
\int_{0}^{T}\left(11 x_{r_{1}}^{*}(t)+50\right) \times\left(t-2 x_{r_{1}}^{*}(t)\right) d t \geq 0, \quad \forall x \in \mathcal{K} .
$$

But, (21) implies that: $2 x_{r_{1}}^{*}(t)=t$; for $t \in[0, T]$ or $x_{r_{1}}^{*}(t)=\frac{t}{2}$. Hence, we also have that $x_{r_{2}}^{*}(t)=\frac{t}{2}$.

Moreover, the equilibrium route costs for $t \in[0, T]$ are given by: $C_{r_{1}}\left(x_{r_{1}}^{*}(t)\right)=5 \frac{1}{2} t+50=$ $C_{r_{2}}\left(x_{r_{2}}^{*}(t)\right)=5 \frac{1}{2} t+50$, and, clearly, equilibrium conditions (10) hold for $\in[0, T]$ a.e.

Assume now that, as depicted in Figure 4, a new link " $e$ ", joining node 2 to node 3 is added to the original network, with cost $c_{e}\left(f_{e}(t)\right)=f_{e}(t)+10$ for $t \in[0, T]$. The addition of this link creates a new route $r_{3}=(a, e, d)$ that is available for the Internet traffic. Assume that the time-varying demand is still given by $d_{w}(t)=t$. Note, that for $t=6$, for example, the original equilibrium flow distribution pattern $x_{r_{1}}(6)=3$ and $x_{r_{2}}(6)=3$ is no longer an equilibrium pattern, since at this level of flow the cost on route $r_{3}, C_{r_{3}}(6)=70$. Hence, the traffic from routes $r_{1}$ and $r_{2}$ would be switched to route $r_{3}$.

The equilibrium flow pattern at time $t=6$ on the new network (which would correspond to the classic Braess paradox in a static network equilibrium setting) is: $x_{r_{1}}^{*}(6)=2, x_{r_{2}}^{*}(6)=2$, $x_{r_{3}}^{*}(6)=2$, with equilibrium link flows: $f_{a}^{*}(6)=4, f_{b}^{*}(6)=2, f_{c}^{*}(6)=2, f_{e}^{*}(6)=2$, $f_{d}^{*}(6)=4$, and with associated equilibrium route costs:

$$
C_{r_{1}}(6)=92, \quad C_{r_{2}}(6)=92, \quad C_{r_{3}}(6)=92 .
$$

Indeed, one can verify that any reallocation of the route flows would yield a higher cost on a route.

Note that, with the route addition, the cost at time $t=6$ increased for every "user" of the network from 83 to 92 without a change in the demand or traffic rate! This is the classical Braess paradox. 


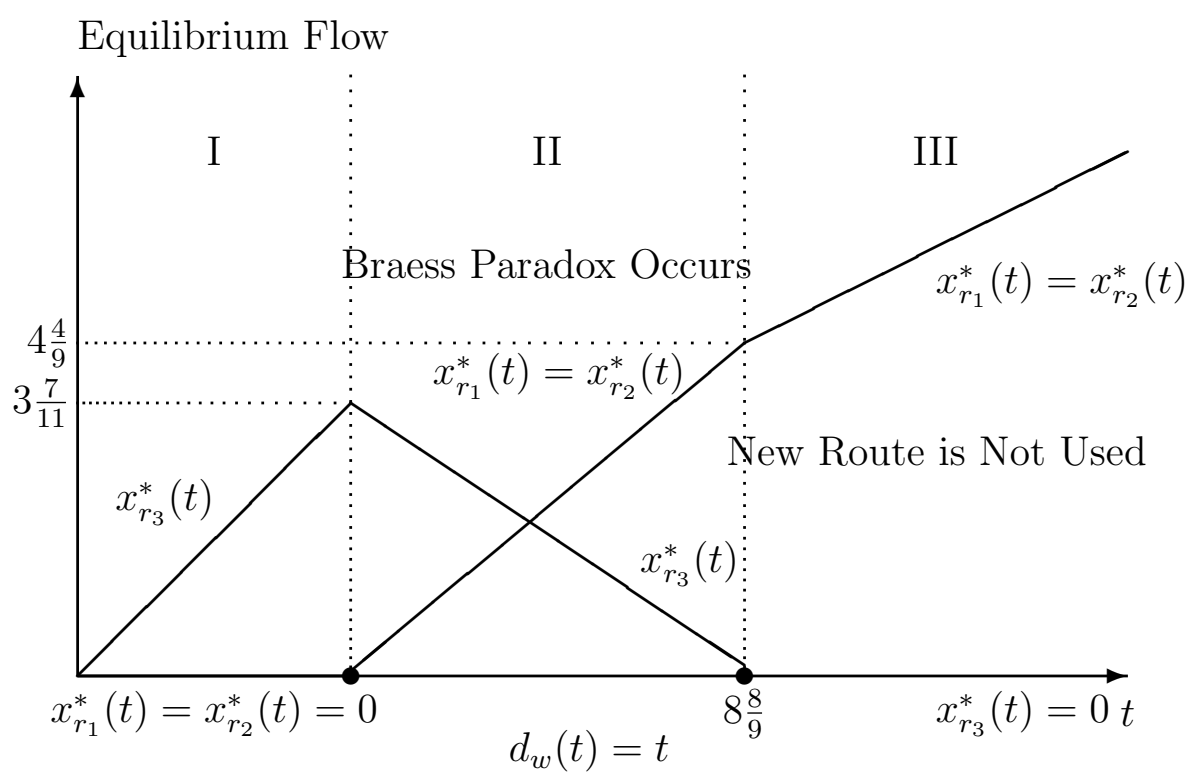

Figure 5: Equilibrium Trajectories of the Braess Network with Time-Dependent Demands

We now recall the solution to the EVI problem (12) for the second network in Figure 4 over the time interval $[0, T]$ which illustrates the time-dependent Braess paradox.

In particular, the solution of the EVI for the second Braess network in Figure 4 with $d_{w}(t)=t$, for $t \in[0, T]$, yields three regimes, denoted by I, II, and III, respectively, and depicted in Figure 4, where: for $d_{w}(t)=t \in\left[0, t_{1}=3 \frac{7}{11}\right]$ (Regime I):

$$
x_{r_{1}}^{*}(t)=x_{r_{2}}^{*}(t)=0, \quad x_{r_{3}}^{*}(t)=d_{w}(t)=t ;
$$

for $d_{w}(t)=t \in\left(t_{1}=3 \frac{7}{11}, 8 \frac{8}{9}\right]$ (Regime II), we have that:

$$
x_{r_{1}}^{*}(t)=x_{r_{2}}^{*}(t)=\frac{11}{13} t-\frac{40}{13}, \quad x_{r_{3}}^{*}(t)=-\frac{9}{13} t+\frac{80}{13} .
$$

Finally, for $d_{w}(t)=t \in\left(t_{2}=8 \frac{8}{9}, T<\infty\right]$ (Regime III), we have that:

$$
x_{r_{1}}^{*}(t)=x_{r_{2}}^{*}(t)=\frac{d_{r_{1}}(t)}{2}=\frac{t}{2}, \quad x_{r_{3}}^{*}(t)=0 .
$$

The curves of equilibria are depicted in Figure 5.

Clearly, one can see from Figure 5 , that in the range $\left(0, t_{1}=3 \frac{7}{11}\right]$, that is, in Regime I (once the demand is positive), only the new route $r_{3}$ would be used. Hence, at a relatively 
low level of demand, up to a value of $3 \frac{7}{11}$, only the new route is used. In the range of demands: $\left(3 \frac{7}{11}, 8 \frac{8}{9}\right]$, that is, Regime II, all three routes are used, and in this range the Braess paradox occurs. Finally, once the demand (recall that $d_{w}(t)=t$ here) exceeds $8 \frac{8}{9}$ and we are in Regime III, then the new route is never used! Thus, the use of an EVI formulation reveals that over time the Braess paradox is even more profound and the addition of a new route may result in the route never being used. Finally, if the demand lies within a particular range, then the addition of a new route may result in everyone being worse off, since it results in higher costs than before the route/link was added to the network.

In particular, the "classical" Braess paradox, in which the addition of the route makes the "travel" cost higher for everyone, always occurs in Regime II. In order to find the minimal demand at which the Braess paradox occurs, we note that in the first network in Figure 4, the demand will always equally distribute itself. Hence, on the original network, the equilibrium flow pattern on each route would be given by $\frac{d_{w}(t)}{2}=\frac{t}{2}$ for $t \in[0, T]$ with a minimal route cost over the horizon being, thus, equal to: $11\left(\frac{t}{2}\right)+50$. Consider now, the second network in Figure 4. We know that in Regime I, only the new route would be used, assuming shortest path routing, with the minimal route cost, hence, being given by the expression in this range of demands as $21 t+10$. Setting now, $11\left(\frac{t}{2}\right)+50=21 t+10$, and solving for $t$, which, is also in this problem equal to the demand, $d_{w}(t)$, yields $t=2 \frac{18}{31}=2.58$. For demand in the range $2.58<d_{w}(t)=t<8 \frac{8}{9}=8.89$, the addition of the new route will result in everyone being worse off. See Figure 6 . 


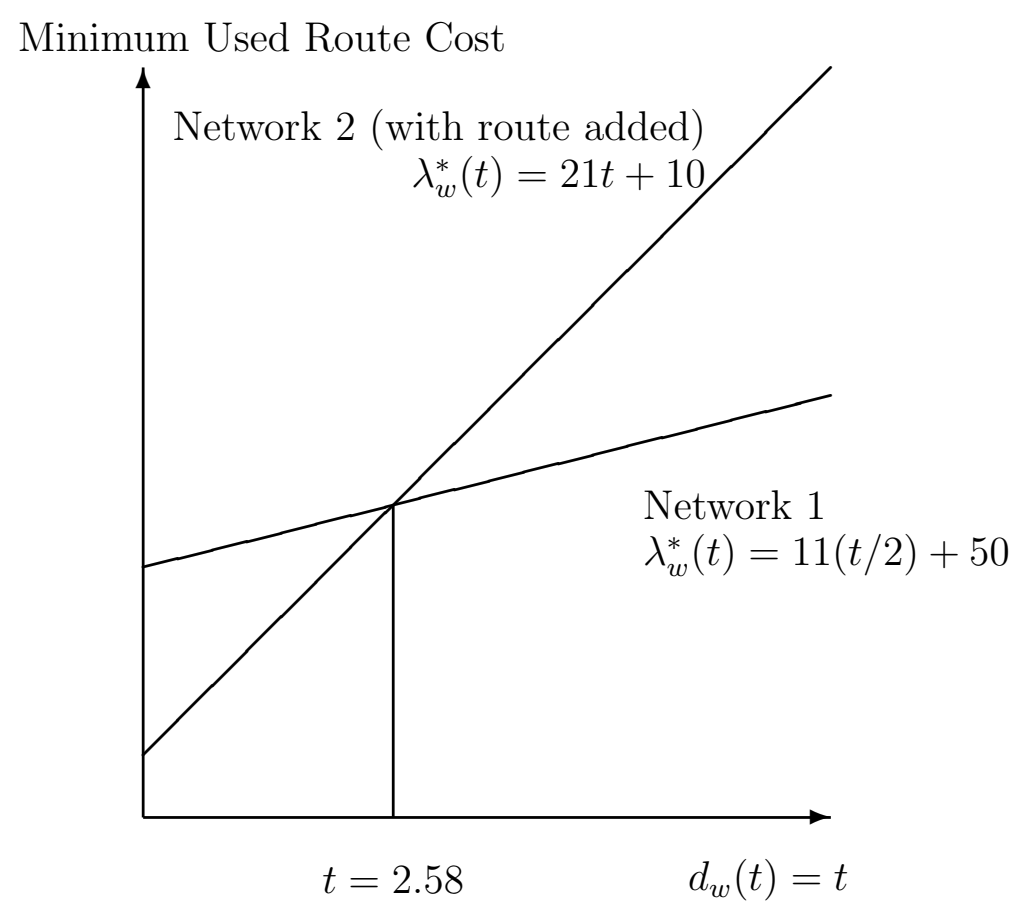

Figure 6: Minimum Used Route Costs for Braess Networks 1 and 2

For both networks in Figure 4, with the associated link and route cost functions, it is easy to verify that the corresponding vector of route costs $C(x)$ is strictly monotone (cf. Nagurney (1993) and Daniele (2006)) in route flows $x$, that is,

$$
\left\langle\left\langle C\left(x^{1}\right)-C\left(x^{2}\right), x^{1}-x^{2}\right\rangle\right\rangle>0, \quad \forall x^{1}, x^{2} \in \mathcal{K}, x^{1} \neq x^{2},
$$

since the Jacobian of the route costs is strictly diagonally dominant at each $t$ and, thus, positive definite. Hence, the corresponding equilibrium route flow solutions $x^{*}(t)$ will be unique.

\section{Network Efficiency of the Dynamic Braess Network and Importance Rankings of Nodes and Links Over the Time Horizon}

Let us now consider the second dynamic Braess network in Figure 4 for $t \in[0,10]$. As shown in Nagurney, Parkes, and Daniele (2007) and recalled above, different routes (and links) 
are used in different demand ranges. Therefore, it is interesting and relevant to study the network efficiency and the importance of the network components over the time horizon. Since in the above example, the demand varies continuously over time, the formula (13) is used to compute the network efficiency. The computations for both (13) and for (17) were done using Matlab (see www.mathworks.com).

The network efficiency $\mathcal{E}(G, d, 10)$ for this dynamic network is 0.5793 . The importance and the rankings of the links and the nodes are summarized in Table 5 and Table 6 , respectively.

Table 5: Importance and Ranking of Links in the Dynamic Braess Network

\begin{tabular}{|c|c|c|}
\hline Link & Importance Value & Importance Ranking \\
\hline$a$ & 0.2604 & 1 \\
\hline$b$ & 0.1784 & 2 \\
\hline$c$ & 0.1784 & 2 \\
\hline$d$ & 0.2604 & 1 \\
\hline$e$ & -0.1341 & 3 \\
\hline
\end{tabular}

Table 6: Importance and Ranking of Nodes in the Dynamic Braess Network

\begin{tabular}{|c|c|c|}
\hline Node & Importance Value & Importance Ranking \\
\hline 1 & 1.0000 & 1 \\
\hline 2 & 0.2604 & 2 \\
\hline 3 & 0.2604 & 2 \\
\hline 4 & 1.0000 & 1 \\
\hline
\end{tabular}

From the above analysis, it is clear which nodes and links are more important in the dynamic Braess network, and, hence, should, in effect, be better protected and secured, in practice, since their elimination results in a more significant drop in network efficiency or performance. Indeed, link $e$ after $t=8 \frac{8}{9}$ is never used and in the range $t \in\left[3 \frac{7}{11}, 8 \frac{8}{9}\right]$ increases the cost, so the fact that link $e$ has a negative importance value makes sense; over time, its removal would, on the average, improve the network efficiency! This analysis also has implications for network design since, over the time horizon, adding/building link $e$ does not make sense. 


\section{Summary and Conclusions}

In this paper, we proposed a network efficiency measure that can be used for dynamic networks, formulated as evolutionary variational inequalities, including the Internet. The network efficiency measure captures costs/latencies on networks with time-varying demands. We provided both a continuous time version of the efficiency measure and a discrete version and then showed that the dynamic network efficiency measure in the case of fixed demands for all the origin/destination pairs over time collapses to the recently proposed measure in Nagurney and Qiang (2007a, b) for transportation (as well as other congested) networks, when such problems are formulated as network equilibrium problems in a static setting. The novelty of the measure lies in that it enables us to assess the network performance without worrying about the connectivity assumption and further, it can be utilized to study the importance of network components under changing demands over the time horizon of interest. To the best of our knowledge, this is the first, rigorous, and well-defined, dynamic network efficiency measure. Such a tool, we expect, will be quite useful for security purposes since the dynamic network itself will be most vulnerable, as measured by the drop in efficiency, if the most important nodes (or links) are removed, due to, for example, structural failures, natural disasters, terrorist attacks, etc.

We illustrated the formalism on three dynamic network examples, including the timedependent Braess paradox. From the results of the importance rankings of individual nodes and links, we can see that when evaluating the vulnerability of a network, all the relevant information regarding the demands, the flows, and the costs over time has to be taken into consideration.

Future research will include the application of the results in this paper to large-scale dynamic networks in telecommunication applications as well as other applications, such as electric power supply chain networks (cf. Nagurney et al. (2007)). Also, we hope to use the proposed network measure to study network robustness and reliability under uncertainty. Finally, it would be very interesting to extend the results in this paper to generalize the measure to the case of price-dependent, time-varying demands, the static version of which was proposed by Qiang and Nagurney (2008). 


\section{Acknowledgments}

The authors are grateful to the four anonymous reviewers for helpful comments and suggestions on an earlier version of this paper.

This research was supported, in part, by NSF Grant No.: IIS-0002647, under the Management of Knowledge Intensive Dynamic Systems (MKIDS) program. The first author also acknowledges support from the John F. Smith Memorial Fund at the University of Massachusetts at Amherst. The support provided is very much appreciated.

\section{References}

Barbagallo, A. (2007), Regularity Results for Time-Dependent Variational Inequalities and Quasi-Variational Inequalities and Application to the Calculation of Dynamic Traffic Network, Mathematical Models and Methods in Applied Sciences 17, 277-304.

Beckmann, M. J.(1967) On the Theory of Traffic Flows in Networks, Traffic Quarterly 21, 109-116.

Beckmann, M. J., McGuire, C. B., and Winsten, C. B. (1956) Studies in the Economics of Transportation, Yale University Press, New Haven, Connecticut.

Bertsekas, D. P., and Gallager, R. G. (1992) Data Networks 2nd Ed., Prentice-Hall, Englewood Cliffs, New Jersey.

Bertsekas, D. P., and Tsitsiklis, J. N. (1997) Parallel and Distributed Computation: Numerical Methods, Prentice-Hall, Englewood Cliffs, New Jersey.

Bienenstock, E. J., and Bonacich, P. (2003) Balancing Efficiency and Vulnerability in Social Networks, in Dynamic Social Network Modeling and Analysis: Workshop Summary and Papers, The National Academy of Sciences, pp. 253-264.

Boyce, D.E., Mahmassani, H. S., and Nagurney, A. (2005) A Retrospective of Beckmann, McGuire, and Winsten's Studies in the Economics of Transportation, Papers in Regional Science 84, 85-103.

Braess, D. (1968) Uber ein Paradoxon aus der Verkehrsplanung, Unternehmensforschung 12, 
$258-268$.

Braess, D., Nagurney, A., and Wakolbinger, T. (2005) On a Paradox of Traffic Planning, Translation of the 1968 Article by Braess, Transportation Science 39, 446-450.

Cantor, D. G., and Gerla, M. (1974) Optimal Routing in a Packet-Switched Computer Network, IEEE Transactions on Computers 23, 1062-1069.

Cojocaru, M.-G., Daniele, P., and Nagurney, A. (2005) Projected Dynamical Systems and Evolutionary Variational Inequalities, Journal of Optimization Theory and Applications 27, $1-15$.

Cojocaru, M.-G., Daniele, P., and Nagurney, A. (2006) Double-Layered Dynamics: A Unified Theory of Projected Dynamical Systems and Evolutionary Variational Inequalities, European Journal of Operational Research 175, 494-507.

Dafermos, S. (1980) Traffic Equilibrium and Variational Inequalities, Transportation Science 14, $42-54$.

Daganzo, C. F. (1977a) On the Traffic Assignment Problem with Flow Dependent Costs-I, Transportation Research 11, 433-437.

Daganzo, C. F. (1977b) On the Traffic Assignment Problem with Flow Dependent Costs-II, Transportation Research 11, 439-441.

Dafermos, S. C., and Sparrow, F. T. (1969) The Traffic Assignment Problem for a General Network, Journal of Research of the National Bureau of Standards 73B, 91-118.

Daniele, P. (2003) Evolutionary Variational Inequalities and Economic Models for Demand Supply Markets, Mathematical Models and Methods in Applied Sciences 4, 471-489.

Daniele, P. (2004) Time-Dependent Spatial Price Equilibrium Problem: Existence and Stability Results for the Quantity Formulation Model, Journal of Global Optimization 28, 283295.

Daniele, P. (2006) Dynamic Networks and Evolutionary Variational Inequalities, Edward Elgar Publishing, Cheltenham, England. 
Daniele, P., Maugeri, A., and Oettli, W. (1999) Time-Dependent Variational Inequalities, Journal of Optimization Theory and its Applications 103, 543-555.

Gallager, R. G. (1977) A Minimum Delay Routing Algorithm Using Distributed Computation, IEEE Transaction on Communications 25, 73-85.

Jenelius, E., Petersen, T., and Mattsson, L.-G. (2006) Road Network Vulnerability: Identifying Important Links and Exposed Regions, Transportation Research A 40, 537-560.

Kinderlehrer, D., and Stampacchia, G. (1980) An Introduction to Variational Inequalities and their Applications, Academic Press, New York.

Korilis, Y. A., Lazar, A. A., and Orda, A. (1999) Avoiding the Braess Paradox in NonCooperative Networks, Journal of Applied Probability 36, 211-222.

Latora, V., and Marchiori, M. (2001) Efficient Behavior of Small-World Networks, Physical Review Letters 87, (Article No. 198701).

Latora, V., and Marchiori, M. (2003) Economic Small-World Behavior in Weighted Networks, The European Physical Journal B 32, 249-263.

Latora, V., and Marchiori, M. (2004) How the Science of Complex Networks can Help Developing Strategies against Terrorism, Chaos, Solitons and Fractals 20, 69-75.

Nagurney, A. (1993) Network Economics: A Variational Inequality Approach, Kluwer Academic Publishers, Dordrecht, The Netherlands.

Nagurney, A. (2006) Supply Chain Network Economics: Dynamics of Prices, Flows, and Profits, Edward Elgar Publishing, Cheltenham, England.

Nagurney, A., and Dong, J. (2002) Supernetworks: Decision-Making for the Information Age, Edward Elgar Publishing, Cheltenham, England.

Nagurney, A., Liu, Z., Cojocaru, M. -G., and Daniele, P. (2007) Static and Dynamic Transportation Network Equilibrium Reformulations of Electric Power Supply Chain Networks with Known Demands, Transportation Research E 43, 624-646.

Nagurney, A., Parkes, D., and Daniele, P. (2007) The Internet, Evolutionary Variational 
Inequalities, and the Time-Dependent Braess Paradox, Computational Management Science 4, 355-375.

Nagurney, A., and Qiang, Q. (2007a) A Transportation Network Efficiency Measure that Captures Flows, Behavior, and Costs with Applications to Network Component Importance Identification and Vulnerability, Proceedings of the 18th Annual POMS Conference, May 2007, Dallas, Texas.

Nagurney, A., and Qiang, Q. (2007b) A Network Efficiency Measure for Congested Networks, Europhysics Letters 79, 38005, p1-p5.

Nagurney, A., and Siokos, S. (1997) Financial Networks: Statics and Dynamics, Springer Verlag, Heidelberg, Germany.

Pas, E. I., and Principio, S. L. (1997), Braess Paradox: Some New Insights, Transportation Research B 31, 265-276.

Patriksson, M. (1994) The Traffic Assignment Problem, VSP, Utrecht, The Netherlands.

Qiang, Q., and Nagurney, A. (2008), A Unified Network Performance Measure with Importance Identification and the Ranking of Network Components, Optimization Letters 2, 127-142.

Ran, B., and Boyce, D. E. (1996) Modeling Dynamic Transportation Networks, Springer Verlag, Berlin, Germany.

Resende, M. G. C., and Pardalos, P. M., Editors (2006) Handbook of Optimization in Telecommunications, Springer Science and Business Media, New York.

Roughgarden, T. (2005) Selfish Routing and the Price of Anarchy, MIT Press, Cambridge, Massachusetts.

Smith, M. (1979) Existence, Uniqueness, and Stability of Traffic Equilibria, Transportation Research B 13, 259-304.

Wardrop, J. G. (1952) Some Theoretical Aspects of Road Traffic Research, Proceedings of the Institute of Civil Engineers, Part II, pp. 325-378. 